\title{
Some Aspects of Voltage Source Inverter Control
}

\author{
Zbigniew Rymarski ${ }^{1}$, Krzysztof Bernacki ${ }^{1}$, Lukasz Dyga ${ }^{1}$ \\ ${ }^{I}$ Department of Automatic Control, Electronics and Computer Science, Institute of Electronics, \\ Silesian University of Technology, \\ Akademicka St. 16, 44-100 Gliwice, Poland \\ zbigniew.rymarski@polsl.pl
}

\begin{abstract}
The model of a voltage source inverter depends on its output filter. The parameters of this filter can differ significantly from their nominal values due to power losses in the core of the output coil and changes in its inductance. Only Multi-Input-Single-Output control systems can effectively reduce inverter output voltage distortions for a standard nonlinear rectifier RC load. However, their design requires knowledge of the actual values of the inverter parameters. The novelty of the paper is that it connects the designs of two MISO control systems with a method of the automated measurements of the voltage source inverter parameters. The breadboard verification is also included.
\end{abstract}

Index Terms-Inverters; digital control; magnetic materials; system identification.

\section{INTRODUCTION}

The paper presents the problem of using the actual, measured parameters of a voltage source inverter when designing a control system.

The problems with the control of single-phase voltage source inverters predicted for UPS systems have been widely presented, e.g. [1], [2]. However, there are some facts that cannot be omitted. None of the simple Single-Input-SingleOutput (SISO) (Fig. 1) control loops (when only the output voltage is measured) work correctly for a standard nonlinear load as defined by the EN 62040-3 standard. Although more sophisticated multi-loop SISO systems are more efficient in dumping the output voltage distortions, they have many disadvantages. While the double loop SISO control with the repetitive controller in the outer loop and the instantaneous controller in the inner loop appears to be a very good solution [3], it requires that the inner control loop have a flat magnitude plot. In this case, we can easily design a compensator using the Zero-Phase-Error-Tracking Compensation method [4]. We can obtain such a flat magnitude plot using e.g. the Coefficient Diagram Method [5], [6]. A repetitive controller is a harmonics generator [7] in the feedback loop and it dumps all of the harmonic distortions and reduces the steady state error (treated as the

Manuscript received 5 October, 2016; accepted 2 February, 2017.

Krzysztof Bernacki was supported by the Polish National Science Centre, grant no. 2015/17/N/ST7/03720. This work was partially supported by the Polish Ministry of Science and Higher Education funding for statutory activities. The calculations were carried out using the IT infrastructure funded by the GeCONiI project (POIG.02.03.01-24-099/13). zero order harmonic) very efficiently. However, a repetitive controller remembers all of the previous fundamental cycle samples and tries to reduce the remembered distortion even after it no longer exists. A typical approach to a SISO controller is to treat the load current as an independent disturbance. The lower the output impedance $Z_{\text {OUT }}$ of the inverter, the more efficient the dumping of such a disturbance is (Fig. 1).

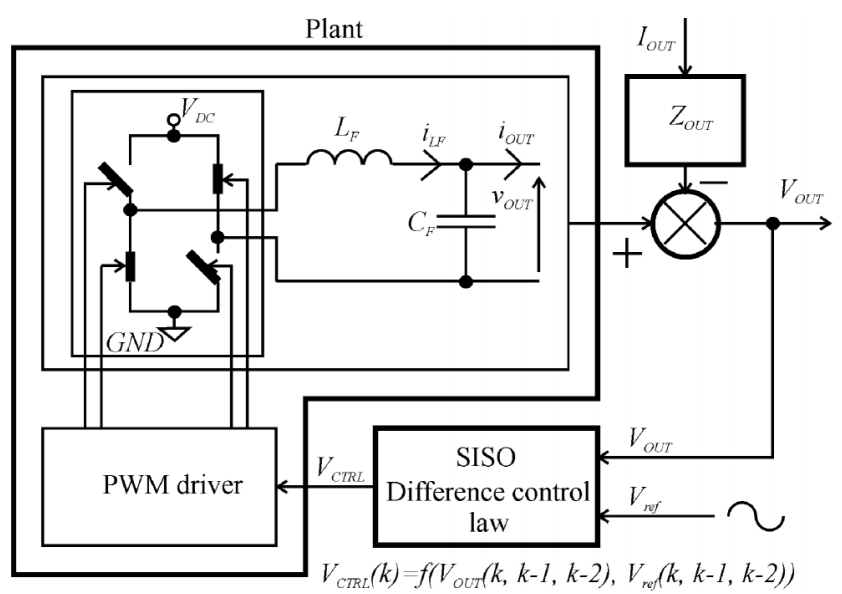

Fig. 1. Single-Input-Single-Output control of a voltage source inverter.

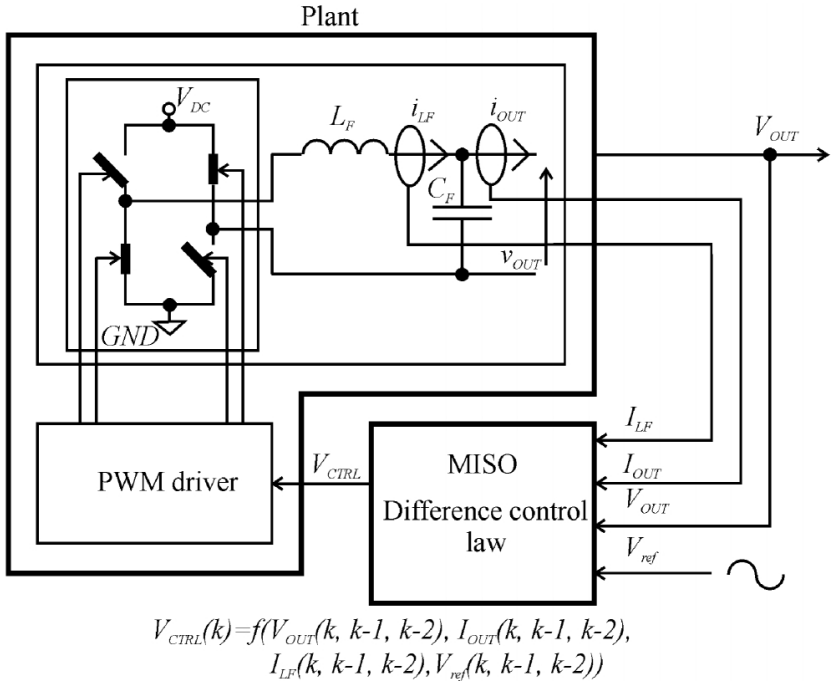

Fig. 2. Multi-Input-Single-Output control of a voltage source inverter.

Therefore, the only solution is to use a Multi-InputSingle-Output controller (Fig. 2). When we measure not only the output voltage but the output current and the inductor 
current (this differs significantly from the output current when the output capacitor has quite a high value) as well, we can reduce the output distortions for a nonlinear load. The other problem is assigning the correct value of the output capacitor. The best approach to this problem is to minimize the reactive power in the output filter inductor and capacitor. However, in such a case, a relatively too low value of the capacitance [8] for the control will be received with the standard switching and sampling frequency being used today - some tens of $\mathrm{kHz}$ (the authors used $f_{c}=25,600 \mathrm{~Hz}$ ). This frequency is too low to reduce the high frequency oscillations of the output voltage after a standard $20 \%$ $100 \%$ step change of the load. The most common solution is to increase the output capacitance [1], [9]. A value of the capacitance that is too high increases the reactive power in the output capacitor and the capacitor current flows through the equivalent serial resistance of the inverter, thus increasing the power losses. Therefore, when the output capacitance is increased, we should control the equivalent serial resistance of the inverter. A meaningful share in this equivalent resistance is caused by power losses in the core of the output filter coil. These losses depend on the frequency and the amplitude of the magnetizing current. The value of the output filter inductance is flexible - the poorer the material, the higher the changes. The complexity of the voltage waveform on the output filter inductor causes the equations given by the core manufacturer [10] to be useless. Only measurements of this inductance and the serial equivalent resistance can provide useful data. However, we should assume the operating point at which these values will be measured. The influence of the switching frequency $f_{c}$ on the serial resistance and inductance is so dominating that we can assume that for the particular switching frequency that is being treated as a parameter, the parameters are not dependent on the frequency.

Two simple MISO control systems will be presented.

The first is a MISO deadbeat controller. A deadbeat controller is designed to set the zero values of the poles of the closed loop system transfer function. In the SISO version, it is very sensitive to every change of the operation point of the inverter and any additional delays in the loop. The dumping of output voltage distortions is not satisfied. The MISO deadbeat version that measures the output current makes the control system independent of the static and dynamic load changes. The design of a MISO deadbeat controller requires knowledge of the state space equations of the inverter. We can use just one of them and assign the output voltage to be equal to the reference voltage in the next sampling period. This method is called a One Sampling Ahead Preview [1]. We should use the measured model including the existing delays in the signal trace. The coefficients of the state space equations depend on the equivalent values of the output filter that will be measured.

The second method is called a Passivity Based Control [9], [11]. It is perfect for power systems because it is based on the system energy flow control (the system should be kept "passive"). In its basic version, the output voltage is not measured but is controlled by means of the inductor current, whose reference depends on the reference voltage. The improved versions [9] use the measurements of the output voltage. In such a case, the parameters of the inverter model are very important.

The parameters of the inverter are measured in an automated laboratory device (Fig. 3) and are compared with the measured Bode plots using a simple continuous inverter model (2).

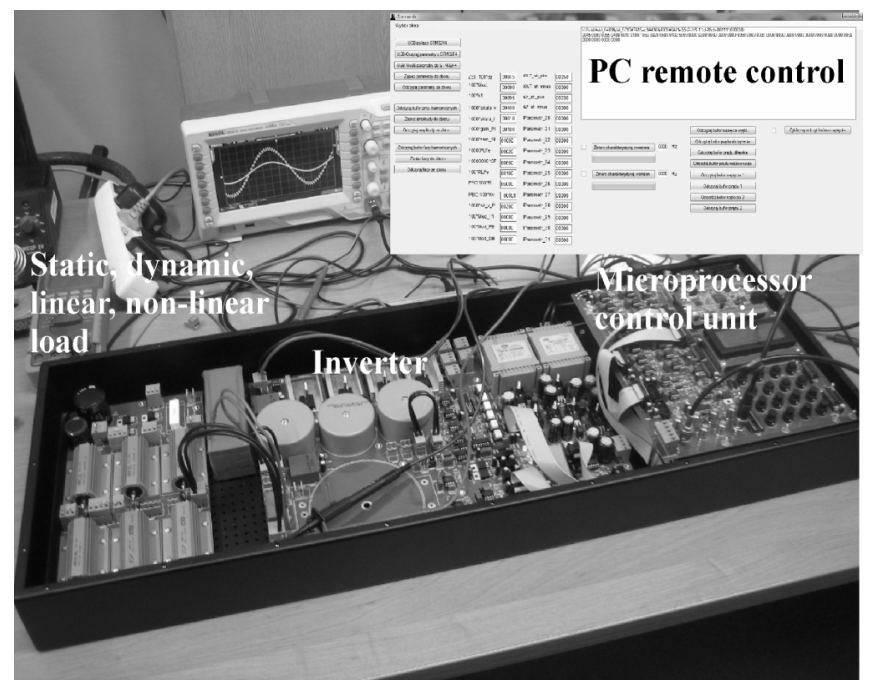

Fig. 3. The laboratory model to measure the actual inverter parameters.

The paper presents the advantages of these two MISO control methods, which are based on the actual measured parameters of the inverter. The method of the inverter Bode plots measurements will also be shown.

\section{Simple Continuous Model OF AN INVERTER}

Our goal was to find the relations (1):

$$
\left\{\begin{array}{l}
L_{F e}=\left.f\left(I_{\text {OUTrms }}\right)\right|_{f_{c}}, \\
R_{\text {LFe }}=\left.f\left(I_{\text {OUTrms }}\right)\right|_{f_{c}}
\end{array} .\right.
$$

The Bode magnitude and phase plots of a simple continuous model of a voltage source inverter with a resistive load (2) are compared with its measurements. In this way, the real parameters $L_{F e}$ and $R_{L F e}$ of the inverter can be calculated. $H(s) \approx 1$ is a transfer function of the PWM modulator:

$$
\begin{aligned}
& K_{C T R L}(s)=\frac{H(s) \frac{1}{L_{F e} C_{F}}}{s^{2}+\left(\frac{R_{L F e}}{L_{F e}}+\frac{1}{R_{L O A D} C_{F}}\right) s+\frac{1}{L_{F e} C_{F}}}, \\
& K_{C T R L}(s) \approx \frac{\omega_{F 0}^{2}}{s^{2}+2 \xi_{F e} \omega_{F 0} s+\omega_{F 0}^{2}},
\end{aligned}
$$

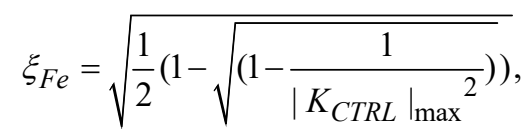

$$
\begin{aligned}
& L_{F e}=\frac{1-2 \xi_{F e}^{2}}{\omega k \max ^{2} C_{F}} \text { for } \xi_{F e}{ }^{2}<0.5 \text {, } \\
& R_{L F e}=\left(2 \xi_{F e} \frac{\omega_{k \max }}{\sqrt{1-2 \xi_{F e}^{2}}}-\frac{1}{R_{L O A D} C_{F}}\right) \frac{1-2 \xi_{F e}^{2}}{\omega_{k \max ^{2} C_{F}}},
\end{aligned}
$$




$$
\text { for } R_{L O A D}>\frac{\sqrt{1-2 \xi_{F e}^{2}}}{2 \xi_{F e} \omega_{k \max } C_{F}} \text { and } \xi_{F e}{ }^{2}<0.5 \text {. }
$$

For the lower dumping coefficients $\xi_{F e}$, the measurement of the serial resistance $R_{L F e}$ is more accurate. Directly designating the transfer function (2) for a high $\xi_{F e}$ (in the presented example for $R_{L O A D} \leq 50 \Omega$ ) is impossible (when we cannot find the maximum of the magnitude plot) and we should use both magnitude and phase plots in the Matlab 'oe' function, which sets the orders [ll 12$]$.

The serial equivalent resistance $R_{L F e}$ includes all of the parasitic serial resistances of the inverter and can be used to calculate power losses in the inverter bridge. However, it strongly depends on the power losses in the core of the filter coil [12]. The equivalent serial resistance of the coil increases significantly over the DC value even for good alloy powder materials such as those used in a breadboard verification using Super-MSS [10]. Changes in the equivalent inductance with the inductor current are low for the alloy powder Super-MSS core material and strong for the iron powder Material Mix. -26 [13].

\section{DISCRETE MODEL OF THE INVERTER}

A discrete model of the inverter is necessary in order to design a MISO deadbeat controller. When solving the continuous state space equations in the switching period and linearizing them [3], [8], we receive the difference state space equations (8). For $\mathbf{x}=\left[v_{\text {OUT }} i_{L F} i_{O U T}\right]^{\mathrm{T}}, \mathrm{i}=1 . .3, \mathrm{j}=1 . .3$ :

$$
\left\{\begin{array}{l}
\mathbf{x}_{k+1}=\mathbf{A}_{\mathrm{D}} \mathbf{x}_{k}+\mathbf{G}_{\mathrm{D}} T_{O N k} \\
\mathbf{A}_{\mathbf{D}}=\left[\phi_{i j}\right] \\
\mathbf{G}_{\mathbf{D}}=\left[g_{i 1}\right]
\end{array}\right.
$$

For the further calculations, we need:

$$
\begin{array}{r}
g_{11}=V_{D C} \omega_{F 0} \sin \left(\omega_{F 0} T_{c} / 2\right) \exp \left(-\xi_{F e} \omega_{F 0} T_{c} / 2\right), \\
\phi_{11}=\left[\cos \left(\omega_{F 0} T_{c}\right)+\xi_{F e} \sin \left(\omega_{F 0} T_{c}\right)\right] \exp \left(-\xi_{F e} \omega_{F 0} T_{c}\right), \\
\phi_{12}=\left(1 / \omega_{F 0} C_{F}\right) \sin \left(\omega_{F 0} T_{c}\right) \exp \left(-\xi_{F e} \omega_{F 0} T_{c}\right), \\
\phi_{13}=-\phi_{12}+R_{L F e}\left(\phi_{11}-1\right), \\
\omega_{F 0}=1 / \sqrt{L_{F e} C_{F e}}, \\
\xi_{F e}=0.5\left(1 / \omega_{F 0}\right)\left(R_{L F e} / L_{F e}\right) .
\end{array}
$$

It can be seen that we do not directly consider the load impedance in the equations because the load current is one of the state variables.

\section{MEASUREMENTS OF THE INVERTER TRANSFER FUNCTION}

The control unit of a device (Fig. 3) to measure the inverter transfer function forces the control voltage of the open loop inverter as a sum fundamental $f_{m}=50 \mathrm{~Hz}$ waveform and excitation waveform that has $n f_{m}$ frequency, whereas in the presented device $n=2 \div 100$

$$
V_{C T R L}(k)=M \times M C I\left[A \sin \left(k \frac{2 \pi}{512}\right)+\right.
$$

$$
\left.+(1-A) \sin \left(n k \frac{2 \pi}{512}\right)\right]
$$

where $M$ is the modulation depth index $\leq 1$, while $M C I$ is the maximum input value of the PWM comparator (in the presented device $M C I=3,280)$. $A$ is the amplitude of the fundamental waveform. For the Super-MSS core material, which has low power losses, it was equal to 0.95 . We store the inverter output voltage $V_{O U T}$ for the particular $V_{C T R L}$ and analyse its spectrum using the MATLAB "fft" function. We cannot directly compare the output in volts and the input in units of the PWM comparator to get the transfer function. The only solution is to measure the magnitude and phase of the excitation harmonics relative to the fundamental harmonic (14). It was assigned that the output for a $50 \mathrm{~Hz}$ waveform had a $0 \mathrm{~dB}$ magnitude and a $0 \mathrm{deg}$ phase

$$
\begin{gathered}
K_{C T R L}\left(j 2 \pi f_{n}\right)= \\
=\frac{\left|V_{\text {OUT }}\left(f_{n}\right)\right| /\left|V_{\text {OUT }}\left(f_{m}\right)\right|}{\left|V_{C T R L}\left(f_{n}\right)\right| /\left|V_{C T R L}\left(f_{m}\right)\right|} \exp \left(j \left\{\left[\operatorname { a r g } \left(V_{\text {OUT }}\left(f_{n}\right)-\right.\right.\right.\right. \\
-\arg \left(V_{\text {OUT }}\left(f_{m}\right)\right]-\left[\arg \left(V_{C T R L}\left(f_{n}\right)-\arg \left(V_{C T R L}\left(f_{m}\right)\right]\right\}\right) .
\end{gathered}
$$

The "row" magnitude and phase Bode plots should be corrected by subtracting the initially measured magnitude and the phase of the measuring channel magnitude and phase from them (Fig. 4). We can calculate the equivalent inductance and serial resistance in the function of the rms value of the inductor current, which is the sum of the load current and the output capacitor current. The relations (1) should be identified for a particular inverter.

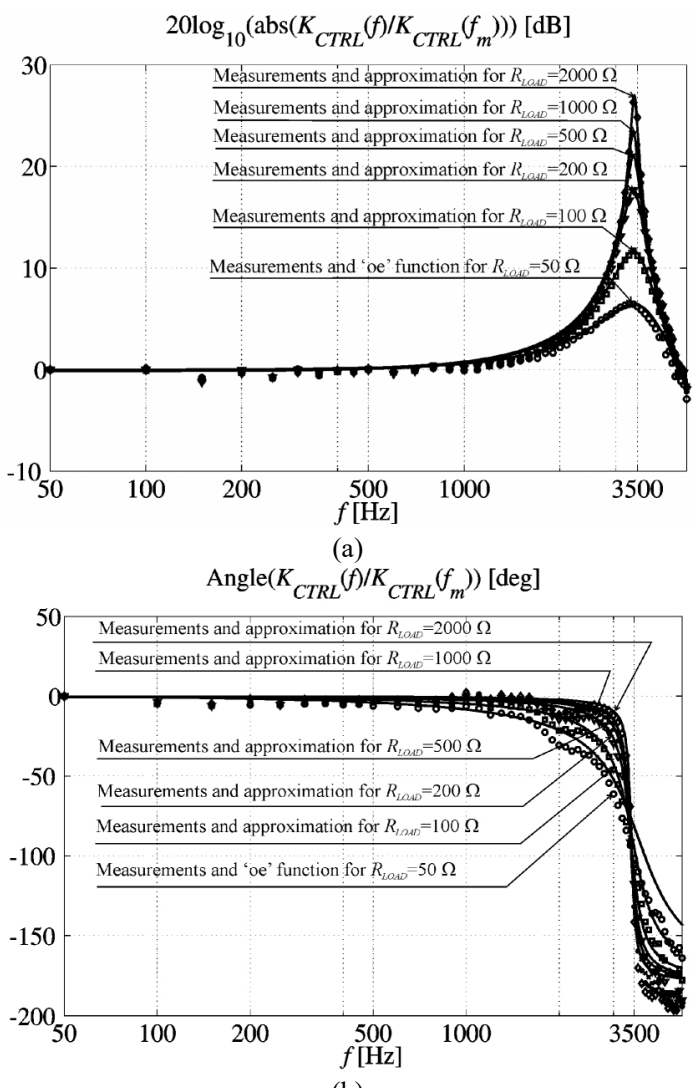

(b)

Fig. 4. Measured and approximated: a) magnitude and; b) phase Bode plots of the laboratory model (for Super-MSS material, $L_{F n o m}=2 \mathrm{mH}$, $\left.C_{F}=1 \mu \mathrm{F}\right)$. 


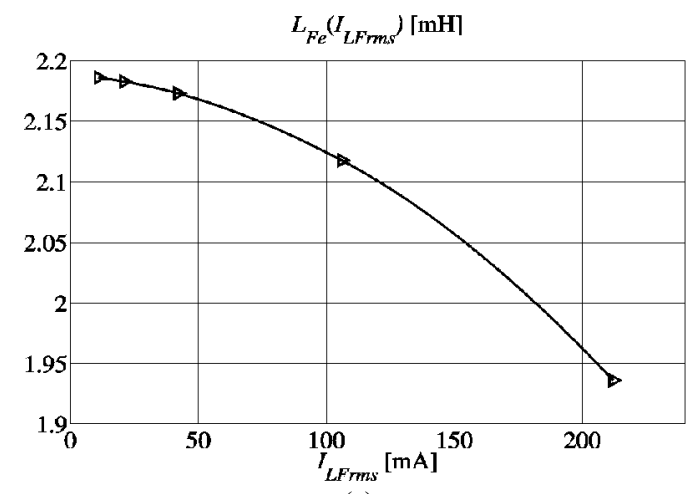

(a)

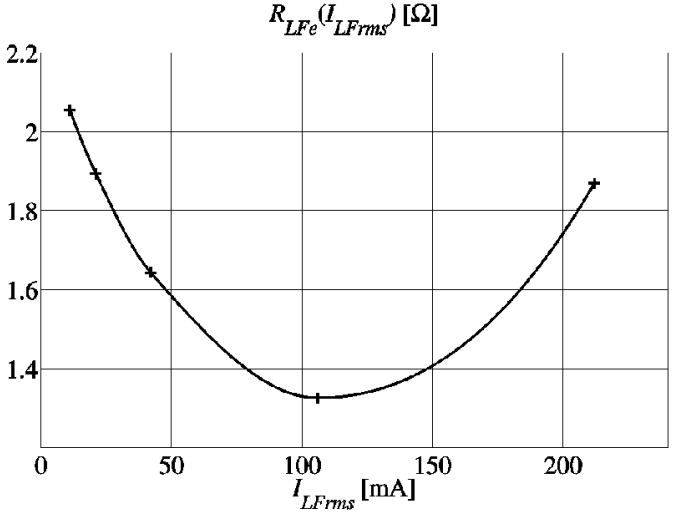

(b)

Fig. 5. The equivalent: a) inductance $L_{F e}\left(I_{L F r m s}\right)$ and; b) the equivalent serial resistance $R_{L F e}\left(I_{L F r m s}\right)$ that were calculated from the Bode plots from Fig. 4.

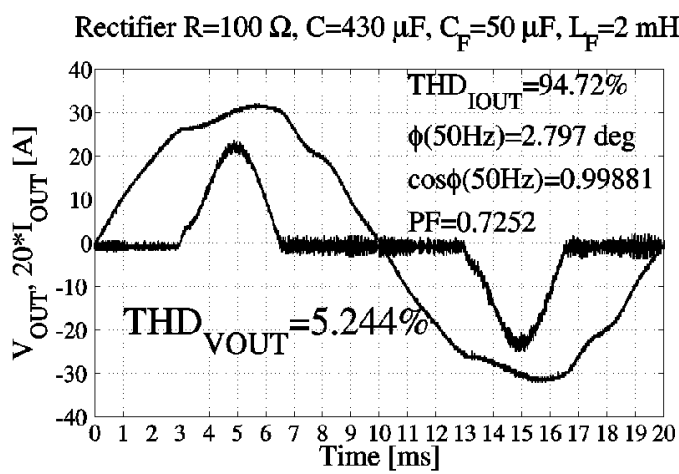

(a)

$\mathrm{V}_{\text {ouT }}{ }^{-\mathrm{h}_{1}}\left(\mathrm{~V}_{\text {ouT }}\right)$, Step load change $45 / 500 \Omega, \mathrm{C}_{\mathrm{F}}=50 \mu \mathrm{F}$

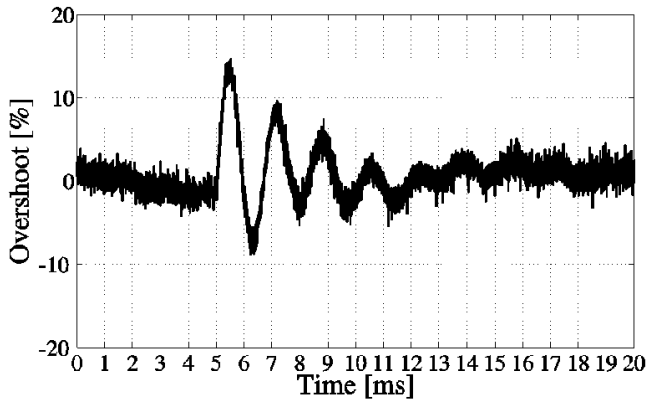

(b)

Fig. 6. The output voltage $T H D$ for: a) the rectifier $R C(100 \Omega / 430 \mu \mathrm{F})$ load $(P F=0.7)$ and; b) the output voltage overshoot for the step load change $\left(R_{L O A D}=45 / 500 \Omega\right)$ for $L_{F n o m}=2 \mathrm{mH}$ and $C_{F}=50 \mu \mathrm{F}$ without feedback.

\section{MISO-DEADBEAT CONTROL}

We will use the One Sampling Ahead Preview Controller:

$$
v_{O U T}(k+1)=v_{r e f}(k+1) \text {. }
$$

The difference control law (18) is calculated from the first state space equation.

$$
\begin{gathered}
V_{C T R L}(k)=\frac{V_{D C}}{g_{11} T_{c}}\left[v_{R E F}(k+1)-\right. \\
-\phi_{11} k_{v_{-} s c a l e} \times v_{O U T}(k)-\phi_{12} k_{i} \text { scale } \times i_{L F}(k)- \\
\left.-\phi_{13} k_{i_{-}} \text {scale } \times i_{\text {OUT }}(k)\right] .
\end{gathered}
$$

The voltage $V_{\text {CTRL }}(k)$ is the input voltage of the PWM modulator (Fig. 2) and is expressed in units of the PWM comparator (the range is from 0 to 3,280 ). We should normalize the real values of the voltage and currents measured with an $\mathrm{AD}$ converter using scaling factors. The coefficients $\varphi_{11}, \varphi_{12}, \varphi_{13}, g_{11}$ are functions of $L_{F e}\left(I_{L F r m s}\right)$ and $R_{L F e}\left(I_{L F r m s}\right)$. The values $L_{F e}$ and $R_{L F e}$ for $I_{L F}=100 \mathrm{~mA}$ were used for the breadboard verification. Figure 7 presents the measurements of the experimental inverter output voltage for the MISO-deadbeat control for the standard loads. Figure 7, (THD VOUT, overshoot) should be compared with Fig. 6.

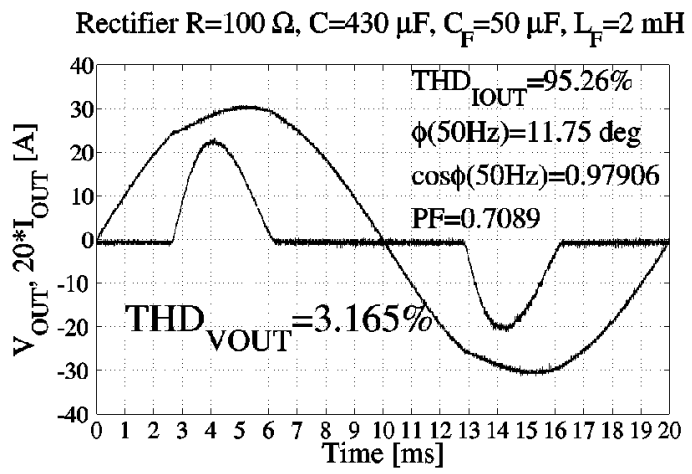

(a)

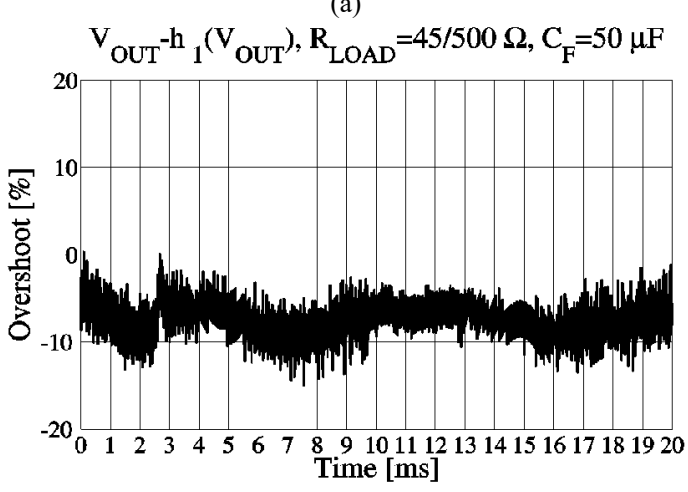

(b)

Fig. 7. The output voltage $T H D$ for: a) the rectifier $R C(100 \Omega / 430 \mu \mathrm{F})$ load $(P F=0.7)$ and; b) the output voltage overshoot for the step load change $\left(R_{L O A D}=45 / 500 \Omega\right)$ for $L_{F}(100 \mathrm{~mA})$ and $C_{F}=50 \mu \mathrm{F}$ for the MISOdeadbeat.

\section{MISO-PASSIVITY BASED CONTROL}

The PBC design involves energy shaping in order to maintain the energy flow of the inverter as well as the damping injection [9]. Although the inverter is described by state space equations, the state variables $\left(v_{O U T}, i_{L F}\right)$ are presented as the sum of their reference value and error. An additional dissipative term is added to both sides of the 
equation that describes the state variable errors on its left side and their references on the right side. The expression describing the error dynamics should be equal to zero and therefore the expression describing the variable references and dissipative term is set to zero. In this way, the difference control law is created (19), (20)

$$
\begin{gathered}
V_{C T R L}(k)=L_{F e}\left[i_{\text {LFref }}(k)-i_{\text {LFref }}(k-1)\right] / T_{c}+ \\
+R_{L F e} i_{\text {LFref }}+v_{\text {Oref }}-R_{i}\left(k_{i_{-} s c a l e} \times i_{L F}-i_{\text {LFref }}\right) .
\end{gathered}
$$

Where reference inductor current is (20)

$$
i_{\text {LFref }}=C_{F} \frac{\left[v_{\text {Oref }}(k)-v_{\text {Oref }}(k-1)\right]}{T_{c}}+k_{i_{-} \text {scale }} \times i_{\text {OUT }} .
$$

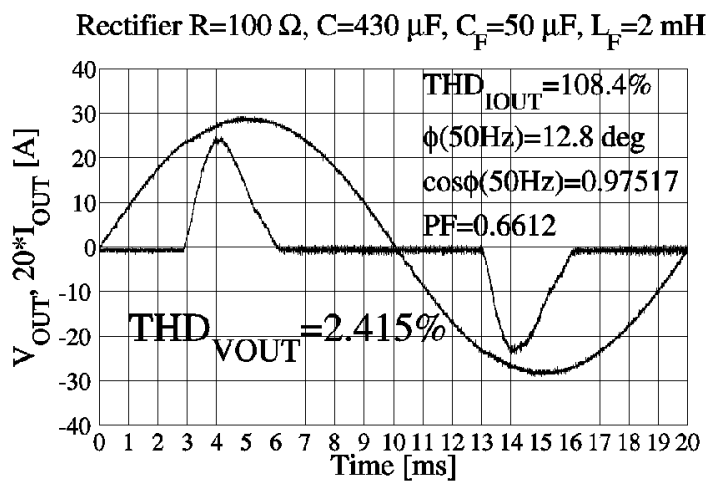

(a)

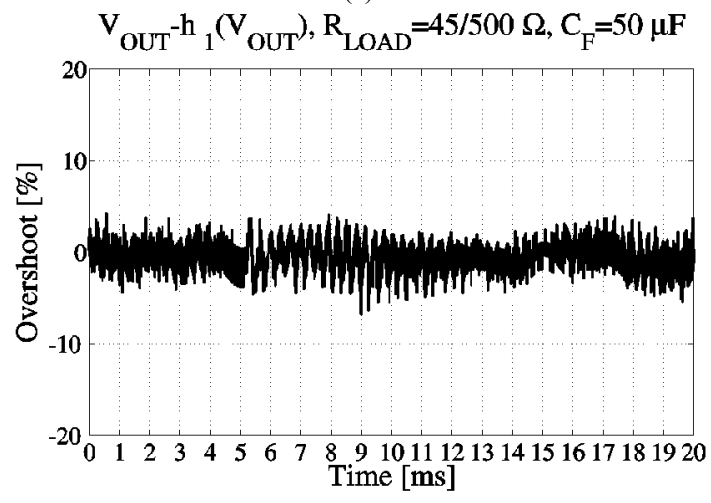

(b)

Fig. 8. The output voltage $T H D$ for: a) the rectifier $R C(100 \Omega / 430 \mu \mathrm{F})$ load $(P F=0.7)$ and; $b)$ the output voltage overshoot for the step load change $\left(R_{L O A D}=45 / 500 \Omega\right)$ for $L_{F}(100 \mathrm{~mA}), C_{F}=50 \mu \mathrm{F}$, MISO-PBC with $R_{i}=50 \Omega$.

The output voltage is controlled by the inductor current [9]. Figure 8 presents the measurements of the experimental inverter output voltage for a MISO-PBC control for the standard loads and should be compared with Fig. 6 .

\section{CONCLUSIONS}

Efficiently dumping the distortions of the output voltage for the standard loads can be done using the actual parameters of the inverter. Only in this manner are we able to significantly lower the THD and overshoots of the output voltage (Fig. 7, Fig. 8 vs. Fig. 6). For a filter coil core that is made of a worse material (e.g. iron-powder Material Mix. 26 [13]), the dependence of the parameters on the load current and the switching frequency is greater. For an alloy powder (e.g. Super-MSS [10]), the changes of the output filter inductance are much lower; however, its equivalent serial resistance is much higher than the DC resistance. The measurement method and the breadboard verification of MISO control loops were presented.

\section{REFERENCES}

[1] L. Ben-Brahim, T. Yokoyama, A. Kawamura, "Digital control for UPS inverters", in Proc. PEDS 2003, vol. 2, pp. 1252-1257. [Online]. Available: http://dx.doi.org/10.1109/PEDS.2003.128315

[2] X. Wang, P. C. Loh, F. Blaabjerg, "Stability analysis and controller synthesis for single-loop voltage-controlled VSIs", IEEE Trans. on Power Electronics, 2016. [Online]. Available: http://dx.doi.org/ 10.1109/TPEL.2016.2632065

[3] C. Rech, H. Pinheiro, H. A. Grundling, H. L Hey, J. R. Pinheiro, "Comparison of digital control techniques with repetitive integral action for low cost PWM inverters", IEEE Trans. on Power Electron., vol. 18, vol. 1/2, pp. 401-410, 2003. [Online]. Available: http://dx.doi.org/10.1109/TPEL.2002.807094

[4] Zhao Gangling, Miao Guan, Yong Wang, "Application of repetitive control for aeronautical static inverter", in Proc. 2nd IEEE Conf. Industrial Electronics and Applications 2007, 2007, pp. 121-125. [Online]. Available: http://dx.doi.org/10.1109/ICIEA.2007.4318382

[5] S. Manabe, "Importance of coefficient diagram in polynomial method", in Proc. 42nd IEEE Conf. Decision and Control, vol. 4, 2003, pp. 3489-3494, [Online]. Available: http://dx.doi.org/ 10.1109/CDC.2003.1271687

[6] J. P. Coelho, T. M. Pinho, J. Boaventura-Cunha, "Controller system design using the coefficient diagram method", Arabian Journal for Science and Engineering, vol. 41, no. 9, pp 3663-3681, 2016. [Online]. Available: http://dx.doi.org/10.1007/s13369-016-2235-y

[7] F. L. Luo, H. Ye, M. Rashid, Digital power electronics and applications. Elsevier Academic Press: USA, 2006.

[8] Z. Rymarski, "The discrete model of the power stage of the voltage source inverter for UPS", Int. Journal of Electronics, vol. 98, no. 10, pp. 1291-1304, 2011. [Online]. Available: http://dx.doi.org/ 10.1080/00207217.2011.589736

[9] H. Komurcugil, "Improved passivity-based control method and its robustness analysis for single-phase uninterruptible power supply inverters", IET Power Electron., vol. 8, no. 8, 2015, pp. 1558-1570. [Online]. Available: http://dx.doi.org/10.1049/iet-pel.2014.0706

[10] Micrometals Arnold Powder Cores 2012 Catalogue, January 2012, Micrometals Inc. 2012.

[11] F. M. Serra, C. H. De Angelo, D. G. Forchetti, "IDA-PBC control of a DC-AC converter for sinusoidal three-phase voltage generation", International Journal of Electronics, vol. 104, no. 1, 2017, pp. 93110. [Online]. Available: http://dx.doi.org/10.1080/00207217 .2016 .1191087

[12] G. Bertotti, "General properties of power losses in soft ferromagnetic materials", IEEE Trans. Magnetics, vol. 24, no. 1, pp. 621-630, 1988. [Online]. Available: http://dx.doi.org/10.1109/20.43994

[13] Z. Rymarski, K. Bernacki, "Different approaches to modelling singlephase voltage source inverters for uninterruptible power supply systems", IET Power Electronics, vol. 9, no. 7, pp. 1513-1520, 2016 [Online]. Available: http://dx.doi.org/10.1049/iet-pel.2015.0142 